\section{Relationship between nuclear DNA-content of sperm cells and the timing of events in the cell cycle of Brassica campestris L. (Brassicaceae)}

\author{
Hua Deng \\ School of Life Sciences, Xiamen \\ University, Centre for Computational \\ Systems Biology, Fudan University, \\ Shanghai, China
}

\begin{abstract}
In this work we conducted a quantitative analysis of the nuclear DNA-content of developing sperm cells of the plant Brassica campestris $\mathrm{L}$. The sperm cells were in young pollen grain, mature pollen grain and pollen tubes. When generative cells, at the pre-anthesis stage, split into two sperm cells, we have established that the newly-formed sperm cells begin to synthesize nuclear DNA in developing pollen grain of $B$. campestris. We measured this DNA-content during the development of sperm cells. The results indicate that during development, sperm cells of $B$. campestris have passed the $G_{1}$ phase of the cell cycle and entered the $S$ phase, presumably then fusing with egg cells at a level of $2 \mathrm{C}$, as is characteristic of $G_{2}$ type fertilization in angiosperms.
\end{abstract}

\section{Introduction}

In sexual reproduction, the life cycle of higher plants is characterized by an alternation of generations, between a haploid gametophyte phase and a diploid sporophyte phase. The diploid sporophyte forms haploid spores through meiosis. The resulting gametophytes form haploid male gametes and female gametes, which fuse to form diploid sporophytes via fertilization. Therefore, alternation of generations in higher plants includes an alternation in the nuclear phase in their life cycle. Some researchers explored this alternation phenomenon by measuring the nuclear DNA-content of male and female gametes of higher plants. They used a method based on the Feulgen reaction in combination with microspectrofluorometry. The nuclear DNAcontent of gametes that were found in the experiments was reported in terms of Relative Fluorescence Units (RFUs). These were then converted to $\mathrm{C}$ value units, where diploid cells have, by definition, a nuclear DNA-content of 2C. ${ }^{1}$ We note that the Feulgen reaction for nuclear DNA is influenced by the gametic origin of the nuclei and the experimental conditions. ${ }^{2,3}$ In addition, in some plants, the egg cells at anthesis cannot be reliably stained; this is thought to be associated with specific patterns of DNA condensation. ${ }^{4,5}$ As a consequence, the nuclear DNA-content of sperm cells that were estimated, by different authors, was not in agreement. For example, in Hordeum vulgare, Hesemann ${ }^{6}$ reported 1-2C, while D'Amato and colleagues ${ }^{7}$ reported $2 \mathrm{C}$. The unreliable experimental situation and consequent unclear results lead to a decline in research associated with the nuclear DNA-content of gametes from the mid 1970's to the 1990's. However, new DNA fluorescence dyes were subsequently developed. These offered greater sensitivity in measuring the nuclear DNA-content of isolated gamete cells from the higher plants. Mogensen and Holm and Mogensen and colleagues ${ }^{8,9}$ demonstrated that the isolated male and female gametes of barley and maize both fused at the $1 \mathrm{C}$ level. Carmichael and Friedman ${ }^{10}$ measured the nuclear DNA-content of male gametes of Gnetum gnemon (a gymnosperm). The data obtained from experiments confirmed that sperm cells and egg cells fuse at the $2 \mathrm{C}$ level. Friedman ${ }^{4}$ later examined the nuclear DNAcontent of sperm cells in Arabidopsis thaliana and determined that two newly formed sperm cells synthesized nuclear DNA-content within pollen grain prior to anthesis and pollen tube germination. Another pattern was reported in tobacco by Tian and colleagues, ${ }^{11}$ where the nuclear DNA of male and female gametes initiated nuclear DNA synthesis when the pollen tube entered the embryo sac. All these reports suggest that novel and unconventional processes occur during gamete development.

The current work is associated with developmental processes of plant sperm cells. This involves the cell cycle, which plays a key role in the division forms that occur in reproduction. In the case of plant gametes, developmental processes, corresponding to the $\mathrm{G}_{1}, \mathrm{~S}$ and $\mathrm{G}_{2}$ phases of the cell cycle occur. ${ }^{4}$

The work of Carmichael and Friedman ${ }^{10}$ has summarized the relationship between fertilization and the timing of events in the cell cycle in gametes of the plants Gnetum gnemon and Ephedra. According to their research, nuclear fusion of gametes, during fertilization, can be classified into three fundamentally distinct types: i) $G_{1}$ phase nuclear fusion. In this case the resulting zygote has twice the nDNA content of a gamete, i.e., the zygotes have an nDNA level of 2C; ii) S phase nuclear fusion. In this case, the resulting zygote has four times the original nDNA content of a gamete, i.e., the zygotes have an nDNA level of $4 \mathrm{C}$; iii) $\mathrm{G}_{2}$ phase nuclear fusion. In this case both sperm cells and egg cells initially have haploid phenotypes with an nDNA content of $1 \mathrm{C}$ but after develop-
Correspondence: Hua Deng, Centre for Computational Systems Biology, Fudan University, 220 Handan Road, Shanghai, 200433, China.

E-mail: denghua@fudan.edu.cn

Key words: Sexual reproduction; Relative Fluorescence Units; C value units; nuclear fertilization.

Acknowledgements: I would like to thank the Botanical Garden of Xiamen University for providing the experimental materials and the laboratory. I also thank Toni Gossmann and David Waxman for help with the writing of this manuscript.

Conflict of interest: the author declares no potential conflict of interest.

Received for publication: 30 November 2015 .

Revision received: 20 January 2016.

Accepted for publication: 20 January 2016.

This work is licensed under a Creative Commons Attribution-NonCommercial 4.0 International License (CC BY-NC 4.0).

(C) Copyright H. Deng, 2016

Licensee PAGEPress srl, Italy

International Journal of Plant Biology 2016; 7:6336

doi:10.4081/pb.2016.6336

ment they separately double their nDNA content and then undergo fusion.

Sexual reproduction, in all three of these distinct types of gamete fusion, is characterized by the difference of the nDNA content of gametes relative to that of somatic cells.

The Cruciferae plant Brassica campestris $\mathrm{L}$. has tri-cellular type pollen; this includes the two sperm cells and the vegetative cell in a mature pollen grain. ${ }^{12}$ This plant has been investigated, using section and microscope observations, on different aspects of morphological, cytological, and ultrastructural characteristics. ${ }^{13}$ Following the investigation of Liu, ${ }^{13}$ and to the best of the author's knowledge, there is no quantitative research on the relationship between the nuclear DNA-content of gametes and changes of the cell cycle of $B$. campestris. To close this gap, we have used DNA fluorescence dyes and microscope observations to investigate the nuclear DNA-content of developing sperm cells in B. campestris. We have found that quantitative changes in DNAcontent are consistent with events that occur in the cell cycle. This suggests that sperm cells in the plant $B$. campestris have passed the $\mathrm{G}_{1}$ phase of the cell cycle, and entered the $S$ phase, presumably fusing with egg cells at $2 \mathrm{C}$ level, as is characteristic of $\mathrm{G}_{2}$ type fertilization in angiosperms. 


\section{Materials and Methods}

Plants of $B$. campestris were grown in the Botanical Garden of Xiamen University. The seeds were sown in October and flowered in March of the following year. When anthers were approximately $2.5 \mathrm{~mm}$ in length, generative cells of $B$. campestris divided to form two sperm cells. When the anthers reached $3 \mathrm{~mm}$ in length, they were collected and considered as young (pre-anthesis) sperm cells. Anthers, when at $5 \mathrm{~mm}$ in length, were collected and classified as mature (anthesis) sperm cells. Flowers were hand pollinated at anthesis and in order to measure the nuclear DNA-content of sperm cells in pollen tubes, the styles were cut at 5 hours after pollination.

Anthers and styles were chemically fixed in 3:1 ethanol (95\%) to acetic acid for approximately 24 hours and subsequently stored in $70 \%$ ethanol at $4^{\circ} \mathrm{C}$. Fixed anthers and styles were serially sectioned at $8 \mu \mathrm{m}$ using a paraffin technique and mounted on glass slides. After paraffin removal, slides were drenched in a solution containing $0.25 \mu \mathrm{g} / \mathrm{mL} \quad 4^{\prime}, 6$ Diamidino-2-Phenylindole (DAPI) in $0.05 \mathrm{M}$ Trizma buffer (pH 7.2) for 20 minutes. Slides were covered with cover slips, examined and photographed with a microscope (a Leica DMR florescence microscope). All samples were captured under the same illumination strength and parameter settings.

Simple-PCI analysis software (Compix Inc, Lake Oswego, OR, USA) was used to analyze the samples. The RFUs of generative nuclei, immediately prior to nuclear division, were used as a control for the diploidy of sperm nuclei of pollen grain in the experiments (diploidy 2C). However, because sperm cells in pollen tubes have a very different growth environment (compared with sperm cells in pollen grain), the style cell nuclei (diploidy $2 \mathrm{C}$ ) were used as a control for the diploidy of sperm cells in pollen tubes. In this experiment, we determined that these style cells were all in the same stage of development, by testing their fluorescence value (we chose a window of fluorescence levels of width 10 RFUs around the mean value, as an indicator of the stage of the development). Sperm cells in different stages of the developmental phase in pollen grain of B. Campestris were measured and analyzed. There were 80 sperm cells measured in young pollen grain, 80 sperm cells measured in mature pollen grain and 28 sperm cells measured in pollen tubes. These measured numbers, reported in Tables 1 and 2, provide adequate numbers for a T-test Analysis.

In Table 1 we separately measured the nuclear DNA-content of three cell types: generative cells, young sperm cells, and mature sperm cells. Numbers of these three cell types that were analyzed were 60,80 and 80 , respec- tively. The mean RFU value of generative cells was defined as the control value, i.e., corresponding to the $2 \mathrm{C}$ level; the nuclear DNA-content (in units of $\mathrm{C}$ ) of two sperm cells were then calculated by the formula: $\{[R F U$ of sperm cells- RFU of background (paraffin and slide)] / RFU of controls - RFU of background (paraffin and slide)] $\} \times 2$, where the background fluorescence is calculated for an area of paraffin identical to the area of the image of the sperm cell's nucleus.

In Table 2 we measured the nuclear DNAcontent of two cell types: style cells and sperm cells in pollen tubes. The numbers of the two cell types analyzed were 150 and 28 , respectively. The mean RFU of style cells was considering as the control, i.e., corresponding to the $2 \mathrm{C}$ level; the nuclear DNA-content (in units of C) of sperm cells in pollen tubes were calculated according to the formula given in the Caption of Table 1.

Since egg cells of $B$. Campestris could not be stained, fluorescence was not detectable in mature egg cells at anthesis and measurements were not obtained for this. Additionally, we note that generally, vegetative cells stay at the differentiation stage, ${ }^{1}$ and the fluorescence signal of vegetative cells is weak. . $^{41,14,15}$ However, in this experiment, there was no reliable fluorescence signal from the vegetative cells (their fluorescence was found to be strongly abnormal) hence we disregarded the vegetative cells.

\section{Results}

\section{Nuclear DNA-content of sperm cells in pollen grain}

When anthers were approximately $2.5 \mathrm{~mm}$ in length, some generative cells in anthers of B. campestris divided into sperm cells. However, in some anthers, generative cells still maintained their original shape in prophase (Figure 1a,b). These generative cells had completed DNA synthesis and were prepared for division. The nuclear DNA-content of these generative cells was at the level of $2 \mathrm{C}$. The mean RFU of the measured generative cells based on data in Table 1 (involving 60 measurements) was 58.35. This value was taken to be the control for the RFU of sperm cells in pollen grain, and hence was taken to correspond to a DNA-content of $2 \mathrm{C}$. When anthers were approximately $3 \mathrm{~mm}$ in length, pollen grain formed two sperm cells (Figure 1c,d), which exhibited an elongated shape and remained isolated. A total of 80 sperm cells were measured and these had a mean RFU of 35.29. Compared with measurements of the generative nucleus (2C), the nuclear DNAcontent of sperm cells at this stage was $1.22 \mathrm{C}$ (Table 1). On the day prior to anthesis, the anthers reached approximately $5 \mathrm{~mm}$ in length and the size of the pollen grain increased to the mature shape (Figure 1e,f). In this developmental phase, the mean RFU, from 80 sperm cells, was determined to be 39.46 . This value corresponds to a nuclear DNA-content of sperm cells of $1.35 \mathrm{C}$. This means that sperm cells had, on average, increased their nuclear DNA-content by approximately one-third (Table 1). From the figures, it is evident that sperm cells in mature pollen grain exhibit greater fluorescence than sperm cells in young pollen grain (Figure 1d,f).

\section{Nuclear DNA-content of sperm cells in pollen tubes}

Following pollen grain germination, pollen tubes elongated, through the style tissue, until they approached an ovary and entered into the embryo sac. In the bright field of the microscope, the unstained style tissue structure was unclear and it was difficult to discern pollen tubes from style cells (Figure 1g,i). However, in the fluorescence-field, the sperm cells in

Table 1. Nuclear DNA-content of generative cells and sperm cells in different stages of pollen grain.

\begin{tabular}{lccc} 
Cell type & N. measurements & RFU, mean \pm SD & Mean DNA level \\
Generative cells (control) & 60 & $58.35 \pm 10.47$ & $2.00 \mathrm{C}$ \\
Young sperm cells & 80 & $35.29 \pm 6.39$ & $1.22 \mathrm{C}$ \\
\hline Mature sperm cells & 80 & $39.46 \pm 6.82$ & $1.35 \mathrm{C}$ \\
\hline
\end{tabular}

RFU, relative fluorescent unit; SD, standard deviation.

Table 2. Nuclear DNA-content of style cells and sperm cells in pollen tubes.

\begin{tabular}{lccc} 
Cell type & N. measurements & RFU, mean \pm SD & Mean DNA level \\
Style cells (control) & 150 & $114.29 \pm 12.03$ & $2.00 \mathrm{C}$ \\
Sperm cells & 28 & $101.98 \pm 18.28$ & $1.78 \mathrm{C}$ \\
\hline
\end{tabular}

RFU, relative fluorescent unit; SD, standard deviation. 
pollen tubes were distinguishable from style cells due to the different morphology and prominent nuclear fluorescence. In addition, the sperm cells were generally connected (Figure 1h,j), which could serve as a sperm cell identifier. As a consequence, 28 sperm cells (14 pairs) in pollen tubes were measured. Additionally, 150 style cell nuclei were measured, and these were used to establish a nuclear DNA reference value (2C), and resulted in the mean nuclear DNA-content of the 28 sperm cells of $1.78 \mathrm{C}$ (Table 2). These results indicate that the sperm cells continue to synthesize nuclear DNA while travelling through pollen tube tissue. We compared the nuclear DNA-content (in $\mathrm{C}$ units) of sperm cells in different developmental phases. These were: sperm cells in young pollen grain, sperm cells in mature pollen grain and sperm cells in pollen tubes. We concluded from this that the main biological phenomenon of sperm cells, during the developmental process, is nuclear DNA synthesis (Figure 2).

\section{T-test analysis}

Quantitative data (RFUs; available on request) were all analyzed using a $t$-test that compared pairs of datasets namely: i) generative cells and young sperm cells; ii) generative cells and mature sperm cells; iii) style cells and sperm cells in pollen tubes. We assumed that the two groups in i) had the same mean value of the RFUs, similarly the two groups in ii) and the two groups in iii). This was the null hypothesis. The alternate hypothesis was that the mean values were not the same (we used a two sided $t$ test). The data analysis indicated a statistically significant result at the $5 \%$ level with rejection of the null hypothesis. That is, we found that RFUs of generative cells compared with young sperm cells were significantly different; generative cells compared with mature sperm cells were significantly different; sperm cells in pollen tubes compared with style cells were significantly different.

\section{Interpretation of data analysis}

The comparison of RFUs of: i) generative cells and young sperm cells; ii) generative cells and mature sperm cells; iii) style cells and sperm cells in pollen tubes, all showed statistically significant results at the $5 \%$ level. We interpret this as saying that during the developmental phase, sperm cells undergo synthesis of DNA-content. The last time of measurement of RFUs of sperm cells, in these experiments, is when they went through pollen tubes. Compared with the results with the RFUs of style cells, we found significant differences. Based on this, we assume that from the last time the DNA-content of sperm cells is measured in pollen tubes, to the time the sperm cells are released into the embryo sac and prepare for fertilization with egg cells, the

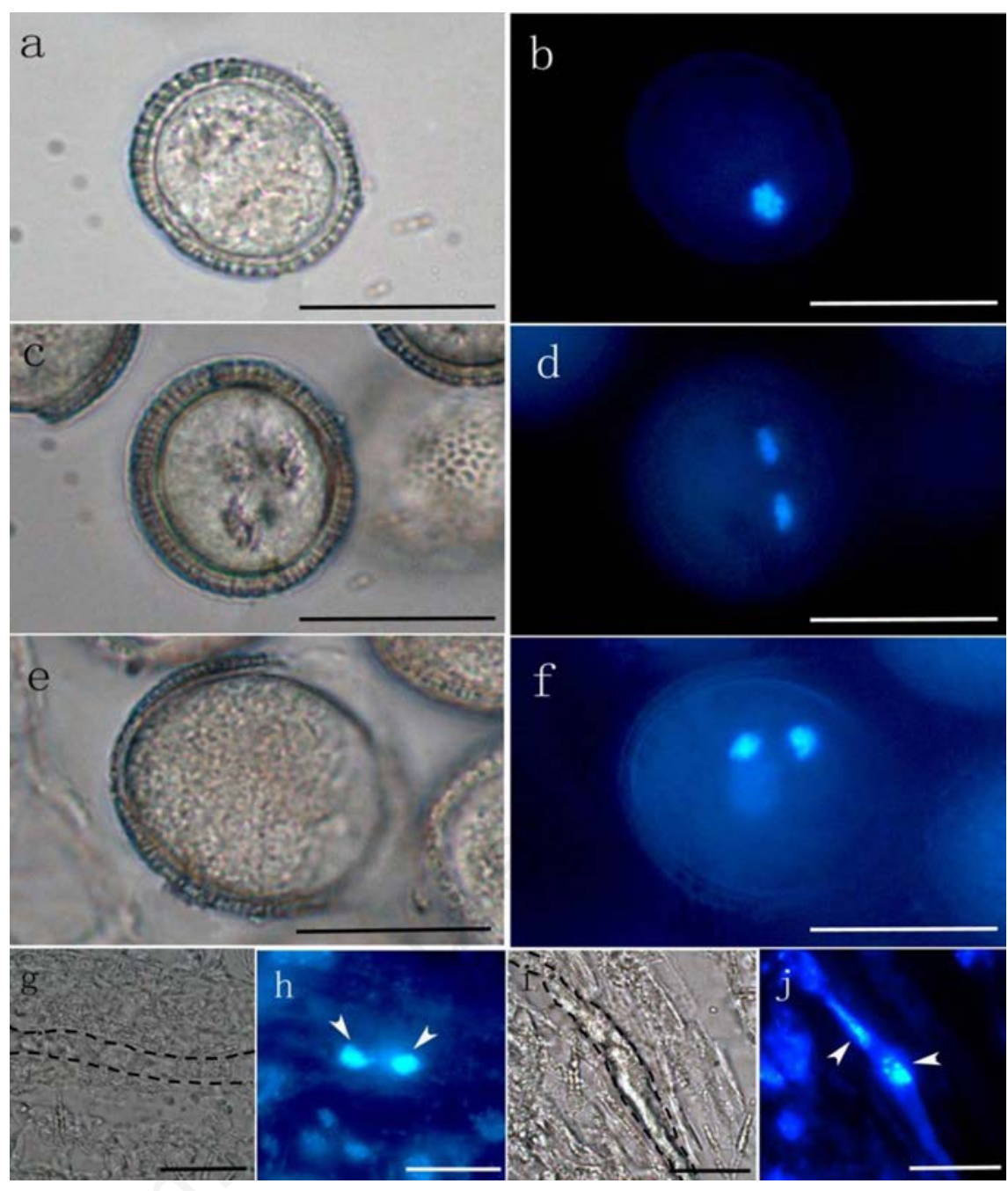

Figure 1. a) A young pollen grain. b) Epifluorescence micrographs of Panel a show a generative cell in prophase. c) A young pollen grain. d) Epifluorescence micrographs of Panel c show two sperm cells in a young pollen grain. e) A mature pollen grain. f) Epifluorescence micrographs of Panel e show two sperm cells in a mature pollen grain. g) Style tissue, under the bright-field of the microscope. The area of the black dashedline indicates the shape of a pollen tube. h) Epifluorescence micrographs of Panel g show two sperm cells in a pollen tube. The two sperm cells are denoted by white arrowheads. i) Bright-field image of style tissue including another pair of sperm cells in a pollen tube. The area of the black dashed-line indicates the shape of a pollen tube. j) Fluorescent image of sperm cells (denoted by white arrowheads) in Panel i. All images in this Figure have been enlarged 750 times and the horizontal bars in the panels represent a distance of $5 \mu \mathrm{m}$.

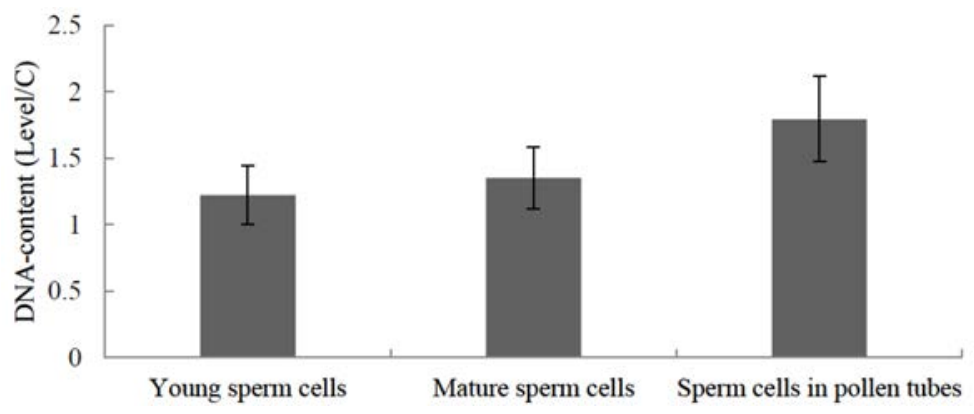

Figure 2. Nuclear DNA-content (level/C) of sperm cells in different developmental stages. This figure shows the increasing trend of the nuclear DNA-content (level/C) of young sperm cells, mature sperm cells and sperm cells in pollen tubes in B. campestris. The error bars of each column indicate one standard deviation of the data above and below the mean value. 
sperm cells continue to synthesize DNA, and finally arrive at a DNA content of $2 \mathrm{C}$.

\section{Discussion and Conclusions}

To begin, let us consider the relationship between this study and taxonomy, in particular, in botany. For traditional botanical taxonomy, researchers focus on solo plants or particular characters, however, the overall explanation of taxonomy is based on an understanding of the relationships among extant species and the Tree of Life. ${ }^{16}$ We note that one aspect of the present study was to investigate whether the $G_{1}, G_{2}$ and $S$ category of nuclear fertilization in plants can act as an indicator of botanical taxonomy. Assuming that plants of the same family all belong to the same class (e.g., $\mathrm{G}_{1}$ ) can the class be used as a characteristic trait of such family? Based on the current study, we can compare two plants $A$. thaliana and B. campestris. Both are Cruciferae plants, with tri-cellular type pollen that are characteristic of $\mathrm{G}_{2}$ plants. Similarly, we can compare tobacco and $L$. barbarum. ${ }^{17}$ Both are Solanaceae plants with bi-cellular type pollen that are characteristic of $\mathrm{G}_{2}$ plants. These two groups of plants are not in the same family, but they are characteristic of $\mathrm{G}_{2}$ plants due to the common trend exhibited by the level of nuclear DNA that is accumulated over the developmental phase. They still, however, have one common point: they all fall under eudicot plants. This suggests that the category of nuclear fertilization in plants cannot be independently used as an indicator of botanical taxonomy. Through the research carried out, this study allows the question mentioned above, namely could the class be used as a characteristic trait of family, to be viewed from a new angle and leads us to ask whether the cell cycle category is related to biological evolution. For all plants measured to date, it is interesting to note that the monocots exhibit the trend of $G_{1}$ fusion while dicots are characteristic of $\mathrm{G}_{2}$. Monocots are a distinct evolutionary lineage arising early in the history of the flowering plants, dat- ing from the early Cretaceous. The basal eudicots and the core eudicots ${ }^{18}$ comprise the dicots and form both ancestral (eudicots) and derived (eudicots) lineages. This suggests some benefit may be gained from a more extensive survey of nuclear fusion of both monocot and dicot plants. The $\mathrm{G}_{1}$ and $\mathrm{G}_{2}$ characters could represent an evolutionary trait among angiosperms, and may constitute an indicator of taxonomy.

\section{References}

1. Hu ShY. Reproductive Biology of Angiosperms. $1^{\text {st }}$ Ed. Beijing: Higher Education Press; 2005. pp 164-70.

2. Shinke N, Ishida MR, Ueda K. A study of the Feulgen reaction of plant cells. Process of International Genetics Symposium, Supplement Volume of Cytologia 1957;15661.

3. Iskida MR. A cytochemical study of nucleic acid in plant cells: V. Nucleic acid of Fulgen negative plants. Cytologia 1961;26:359-71.

4. Friedman WE. Expression of the cell cycle in sperm of Arabidopsis: implications for understanding patterns of gametogenesis and fertilization in plants and other eukaryotes. Development 1999;126:106575 .

5. Zhou C. A study of fertilization events in living embryo sacs from sunflower. Plant Sci 1987;52:147-51.

6. Hesemann CU. Untersuchungen zur Pollenentwicklung und Pollenschlauchbildung bei hoheren Pflanzen. III. DNS-Replikation bei vegetativen und Sperma-Kernen in reifen Pollenkornernvon Gerste. Theor Appl Genet 1973;43:232-41.

7. D'Amato F, Devereux M, Scarascia M, Mugnozza GT. The DNA content of the nuclei of the pollen grain in tobacco and barley. Caryologia 1965;18:377-82.

8. Mogensen HL, Holm PB. Dynamics of nuclear DNA quantities during zygote development in barley. Plant cell
1995;7:487-94.

9. Mogensen HL, Leduc N, Matthys-Rochon E, Dumas C. Nuclear DNA amounts in the egg and zygote of maize (Zea mays L). Planta 1999;197:641-5.

10. Carmichael JS, Friedman WE. Double fertilization in Gnetum gnemon: the relationship between the cell cycle and sexual reproduction. Plant Cell 1995;7:1975-88.

11. Tian HQ, Yuan T, Russell SD. Relationship between double fertilization and the cell cycle in male and female gametes of tobacco. Sexual Plant Reprod 2005;17:243-52.

12. Schmidt A, Schmid MW, Grossniklaus U. Plant germline formation: common concepts and developmental flexibility in sexual and asexual reproduction. Dvelopment 2015;142:229-41.

13. Liu TT. The morphological, cytological and ultrastructural characters of Chinese cabbage-pak-choi (Brassica Campestris L. ssp. Chinensis Makino, syn. Bassica rapa ssp. Chinensis) of flower development. Dissertation 2007. Zhejiang University, China. [Article in Chinese]

14. Hough T, Bernhardt P, Knox RB, Williams EG. Applications of fluorochromes to pollen biology. II. The DNA probes ethidium bromide and Hoechst 33258 in conjunction with the callose-specific aniline blue fluorochrome. Stain Tech 1985;60:155-62.

15. Deng H, Wang YY, Zhu XY, Tian HQ. Generative and sperm cell isolation in Bauhinia blakeana (Fabaceae). Ann Bot Fennici 2012;49:1-6.

16. Cracraft J. The seven great questions of systematic biology: an essential foundation for conservation and the sustainable use of biodiversity. Ann Miss Bot Gard 2002;89:127-44.

17. Deng H, Song YX, Qin K, Tian HQ. DNA Content and cell cycle changes of male and female gametes of Lycium barbarum L. Plant Physiol J 2012;48:869-73. [Article in Chinese]

18. Worberg A, Quandt D, Barniske AM, et al. Phylogeny of basal eudicots: insights from non-coding and rapidly evolving DNA. Org Divers Evol 2007;7:55-77. 